\title{
Personality and fatigue in patients with benign or malignant breast disease
}

\author{
Helen J. Michielsen • Alida F. W. Van der Steeg • \\ Jan A. Roukema • Jolanda De Vries
}

Received: 13 September 2006 / Accepted: 17 January 2007 / Published online: 7 February 2007

(C) Springer-Verlag 2007

\begin{abstract}
Goals of work The aim of the study was to examine the role of five general personality traits in fatigue in a group of patients with breast cancer (BC) and a group with benign breast problems (BBP).

Materials and methods of the 304 participating women, 127 patients had BC and 177 BBP. A fatigue scale was completed before diagnosis and 1,3, and 6 months after diagnosis (benign patients) or surgical treatment $(\mathrm{BC}$ patients). A personality questionnaire (NEO-FFI) and a depression scale (CES-D) were completed before diagnosis. Main results The $\mathrm{BC}$ group was less tired before diagnosis, more tired 1 month after diagnosis, and equally tired 3 and 6 months after diagnosis. In the total group, women were more tired over time when they were more neurotic, less agreeable, or more introverted. After controlling for depressive symptoms, demographics, and medical factors, baseline depressive symptoms $(\beta=0.29, p<0.05)$, neuroti$\operatorname{cism}(\beta=0.29, p<0.05)$, and extraversion $(\beta=-0.25, p<$ $0.05)$ predicted fatigue 6 months later. After also including baseline fatigue, only neuroticism $(\beta=0.22, p<0.05)$ and baseline fatigue $(\beta=0.79, p<0.001)$ predicted fatigue.
\end{abstract}

\footnotetext{
H. J. Michielsen · J. De Vries $(\bowtie)$

Department of Psychology and Health,

Medical Psychology, Tilburg University,

Room P510, P.O. Box 90153,

5000 LE Tilburg, Netherlands

e-mail: j.devries@uvt.nl
}

\author{
A. F. W. Van der Steeg • J. A. Roukema \\ Department of Surgery, St Elisabeth Hospital, \\ P.O. Box 90151, 5000 LC Tilburg, Netherlands \\ J. De Vries \\ St Elisabeth Hospital, \\ P.O. Box 90151, 5000 LC Tilburg, Netherlands
}

Conclusions Personality is more strongly related to fatigue than demographics, the diagnosis cancer, receiving cancer treatment, and baseline depressive symptoms and fatigue. When replicated, screening and treating women who are at risk to experience high levels of fatigue is recommended.

Keywords Breast neoplasm · Fatigue $\cdot$ Personality

\section{Introduction}

Breast cancer $(\mathrm{BC})$ is the most common type of cancer among women. In The Netherlands, $11 \%$ of all women will develop carcinoma in situ or invasive carcinoma of the breast [3]. A common reaction during and after the treatment period is fatigue [1]. In general, $\mathrm{BC}$ patients are not as severely tired as chronic fatigue syndrome patients [24]: their fatigue is mainly mild to moderate [2]. However, fatigue might distress $\mathrm{BC}$ patients [25], reduce their quality of life (QOL) [29], and impair their physical activity [1].

The determinants of fatigue in $\mathrm{BC}$ patients are not yet identified. Physical, cancer- or treatment-related factors such as anemia [7], receiving chemotherapy [16], radiotherapy [25], and/or hormone therapy only partially explain the difference with fatigue in the general population. Regarding the association between fatigue and personality in (breast) cancer patients, inconsistent findings were reported.

Nowadays, there is general agreement about the view that personality, at least for descriptions at a rather global level, can be described adequately in terms of the Big Five dimensions: extraversion, agreeableness, conscientiousness, emotional stability, and openness to experience [see, e.g., 11, 12]. Extraversion reflects the disposition towards cheerfulness, sociability, and high activity. Agreeableness 
represents the inclination towards interpersonal trust and consideration of others. Conscientiousness summarizes the tendency towards persistence, sense of duty, industriousness, organizing, planning, and self-discipline. Emotional stability stands for the tendency to experience no distressing emotions such as fear, guilt, and frustration. Finally, the fifth factor points at a receptive orientation towards varied experiences and ideas [see 9 for a more detailed description of these five basic factors]. In a recent cross-sectional study among disease-free BC patients without depression, neuroticism and introversion were associated with higher levels of fatigue [30]. In another recent cross-sectional study among disease-free cancer patients, only neuroticism was related to fatigue. However, it became non-significant when depression and beliefs were added in the analyses [32]. Furthermore, no association was found between fatigue and neuroticism or optimism in a prospective study among patients with different types of cancer receiving radiotherapy with curative intent [26]. When no additional variables were included in the analyses, extraversion was, and neuroticism was not, related to fatigue in another prospective study among patients with cancers of the breast or prostate undergoing radical radiotherapy [29]. In a 3-month follow-up study by this group, neither neuroticism nor extraversion was associated with fatigue in patients with prostate cancer receiving hormone therapy [28].

Most studies focussed on a small number of personality traits, were cross-sectional, did not measure depression, and/or did not include a comparison group [26, 28-30, 32]. The aim of this 6-month prospective follow-up study was to examine the role of five general personality traits in fatigue in patients having a palpable lump in the breast or being recalled for further examination after an abnormality on a screening mammography.

\section{Materials and methods}

\section{Participants}

Women with a palpable lump in the breast or an abnormality on a screening mammography were referred by their general practitioner to the department of surgery of the outpatient clinics of the St. Elisabeth Hospital, Tilburg (since 1 January, 2002) or Maasland Hospital, Sittard, The Netherlands (since August, 2004). They were asked to participate in a study with a pre- and post-treatment prospective design, focussing on the role of personality in patients' QOL. Thus, the present study is part of a larger study. When the women were asked to participate in the study and completed the first set of questionnaires, it was unknown whether a woman had $\mathrm{BC}$ or a benign breast problem (BBP). Once diagnosis was known, diagnosis was the reference point for subsequent measurement times for benign patients. For BC patients, the reference point was surgical treatment because otherwise follow-up measures would interfere with timing of treatment modalities. Of the eligible 533 women, 183 were later diagnosed with $\mathrm{BC}$, and 305 appeared to have BBP. Exclusion criteria were dementia, history of breast problems, and not being able to read and speak standard Dutch. The study focussed on women with $\mathrm{BC}$ who had a choice between breast conserving treatment (BCT) and modified radical mastectomy (MRM). Women with a breast tumor that was too large to allow women to choose between BCT and MRM were also excluded. Of the BC patients, $122(66.7 \%)$ participated in this study. Of the BBP group, 177 (58.0\%) agreed to participate. The most important reasons for not participating were the length of the questionnaires and the amount of stress the women experienced, which they felt compromised concentration while completing the questionnaires. Women gave written informed consent. Women who participated were younger than the non-participants $[t(1$, $530)=3.45, p<0.01]$. They did not differ on diagnosis .

\section{Measures}

Women completed the questionnaires to assess fatigue four times: before diagnosis (T1) and 1 (T2), 3 (T3), and 6 (T4) months after diagnosis (benign patients) or surgical treatment (BC patients). Personality and depressive symptoms were measured once, before diagnosis. Questionnaires were completed at home or in the hospital, before their check up/visit.

Clinical parameters: tumor size after pathological examination and adjuvant treatment were obtained from the medical records of the included patients.

The Fatigue Assessment Scale (FAS; [19]) is a fatigue questionnaire consisting of ten items: five questions reflecting physical fatigue and five questions assessing mental fatigue. Although these two aspects of fatigue are represented in the questionnaire, the FAS was unidimensional when completed by a Dutch working population and a representative group of the general population $[19,20]$, as well as in sarcoidosis patients $[10,21]$. The unidimensional structure indicates that the FAS total score should be used. The response scale is a 5-point scale ( 1 , never to 5 , always). Scores on the FAS range from 10 to 50 . The psychometric properties are good in Dutch healthy individuals and sarcoidosis patients $[10,20,21]$. In the $\mathrm{BC}$ patients, Cronbach's alpha internal consistency was 0.88 , in the BBP group 0.92. Factor analyses in both groups supported the unidimensional nature of the scale (data not shown).

The Neuroticism-Extraversion-Openness Five Factor Inventory (NEO-FFI; [9] Dutch version [15]) is developed to study an individual's personality. The following five personality factors are tested: neuroticism, extraversion, 
openness, agreeableness, and conscientiousness. The psychometric properties are good $[9,15]$.

The Center for Epidemiological Studies-Depression Scale (CES-D [22]) is a 20-item scale designed to measure the presence and degree of depressive symptoms. It has a 4point response scale. For the Dutch population, reliability and criterion validity appeared to be good [4]. In the present study, the 16-item CES-D as suggested by Schroevers et al. [23] was used because they found it to be a more valid assessment of depressive symptoms in both cancer patients and healthy persons.

\section{Data analysis}

Frequencies were used to present the available demographic, medical, and psychological data. Participants who only completed the first set of questionnaires were compared on age, diagnosis, and kind of treatment with those who also completed the fourth set 6 months later. Differences in fatigue were examined between the BC and the BBP group using $t$ tests for each measurement point. Using repeated measures, fatigue over time was investigated for the $\mathrm{BC}$ and the BBP group, accounting for adjuvant treatment. First, in the $\mathrm{BC}$ patient group, the relationship between clinical parameters and fatigue was examined using $t$ tests (treatment with hormones, chemotherapy, radiotherapy, or not), correlations (hospital stay in days), and analysis of variance (ANOVA; tumor group). Following Cohen [8], absolute correlations between 0.10 and 0.29 are considered small, between 0.30 and 0.49 medium, and between 0.50 and higher as large. Then, the association between demographics and fatigue were studied separately for the $\mathrm{BC}$ and BBP group using $t$ tests (having children or not, having a partner or not, having a paid job or not, age $>60$ or not), correlations (age), and ANOVA (education level). Personality differences between the $\mathrm{BC}$ and $\mathrm{BBP}$ group were examined using a multivariate ANOVA (MANOVA). Eta squared between 0.01 and 0.06 is a small effect by Cohen's definition [8], between 0.06 and 0.13 a moderate effect, and 0.14 or higher a large effect. The stability of fatigue over 6 months was explored, differentiating high and low scorers on the five personality factors in the total group. For this purpose, we divided the total scores of each NEO-five factor into two groups. The first group consisted of the stanines $\leq 5$; the second group of stanines $\geq 6$. Finally, the predictors of scores on fatigue 6 months after diagnosis/ surgical treatment were found using three regression analyses (method enter). In the first analysis, demographic characteristics (block 1), diagnosis (benign or BC), and adjuvant therapy (chemotherapy, radiotherapy, hormone therapy; block 2), and personality (block 3 ) were the independent variables. In the second regression analysis, depressive symptoms were included in the third block and personality in the fourth block. In the final analysis, a fifth block, consisting of baseline fatigue, was incorporated. All $p$ values were two-tailed, and Statistical Package for the Social Sciences (SPSS) 12.0 was used to perform all statistical analyses.

\section{Results}

Demographic and medical data are presented in Table 1. Of the 299 included patients, 122 had BC, and 177 had BBPs. Fifteen women in the BC group and 19 women in the BBP group entered the study less than 6 months earlier. In addition, 6 months after diagnosis (BBP) or surgical treatment (BC), 63 (39.9\%) with a benign diagnosis and 29 (27.1\%) who were diagnosed with BC had dropped out of the study. They did not differ on age, diagnosis, chemotherapy, hormone therapy, radiotherapy, fatigue, and personality from those who completed all questionnaires.

The BC group showed a different fatigue pattern over time from the BBP group [Wilks Lambda $=0.80, F(3,137)=11.55$, $p<0.001$, partial eta squared 0.20 ; see Fig. 1]. They were less tired before diagnosis $[19.2 \pm 6.7,21.6 \pm 8.0$, respectively; $t(1,272)=2.61, p<0.01]$, more tired 1 month after diagnosis $[t(1,215)=2.51, p<0.05]$, and equally tired 3 and 6 months after diagnosis.

In the $\mathrm{BC}$ group, fatigue measured at $\mathrm{T} 2-\mathrm{T} 4$ was not associated with tumor group $(p>0.05)$. At T3, patients who were treated with radiotherapy were more tired $[t(1,189)=$ $2.30, p<0.05]$. No differences for fatigue were found on other measurement points or with chemotherapy or hormone therapy.

After Bonferonni correction, the association between demographics and fatigue in the BC and BBP group was not significant for age (continuous), age $>60$ years, education level, having children, having a paid job, and having a partner. There were no personality differences between the

Table 1 Demographic and medical characteristics of the breast cancer patients and females with a benign breast problem

\begin{tabular}{lll}
\hline $\begin{array}{l}\text { Demographic and medical } \\
\text { characteristics }\end{array}$ & $\begin{array}{l}\text { Breast cancer } \\
(n=122)\end{array}$ & $\begin{array}{l}\text { Benign breast } \\
\text { problem }(n=177)\end{array}$ \\
\hline Age $^{\text {a }}$ & $58.4 \pm 9.5$ & $51.58 \pm 10.8$ \\
Having children & $84(68.9 \%)$ & $92(52.0 \%)$ \\
Having a partner & $94(77.1 \%)$ & $144(81.4 \%)$ \\
$10-14 />14$ years of & $42(34.4 \%) / 16$ & $70(39.5 \%) / 30$ \\
education & $(13.1 \%)$ & $(16.9 \%)$ \\
Paid work & $41(33.6 \%)$ & $97(54.8 \%)$ \\
Tumor $<1 / 1-3 />3 \mathrm{~cm}$ & $24 / 79 / 19$ & \\
Mastectomy/breast saving/? & $73 / 49$ & \\
Chemotherapy & 32 & \\
Hormone therapy & 47 & \\
Radiotherapy & 58 & \\
\hline
\end{tabular}

${ }^{\mathrm{a}}$ Data are expressed as mean $\pm \mathrm{SD}$. 
Fig. 1 Course of fatigue over 6 months in women with benign breast problems or cancer
Fatigue

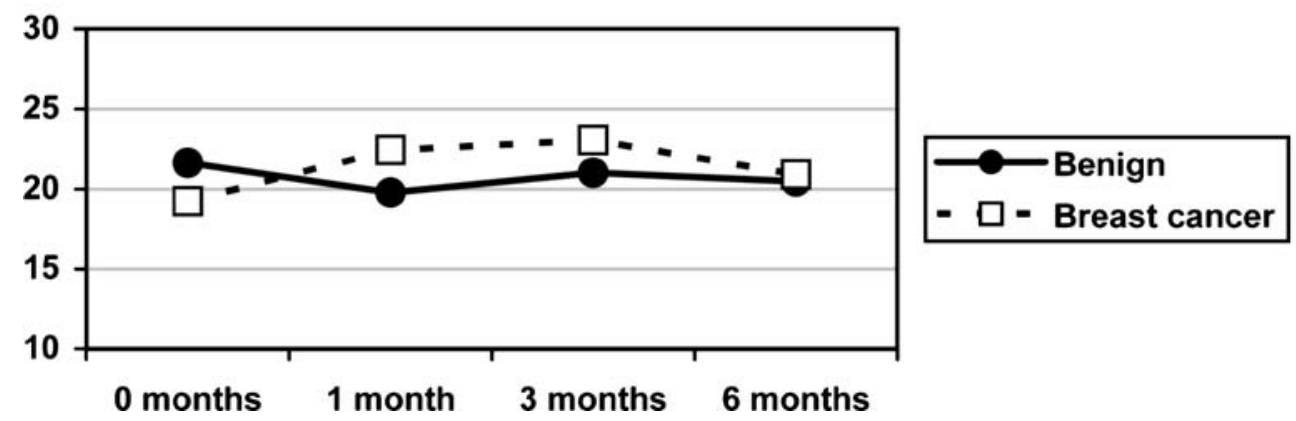

$\mathrm{BC}$ and BBP group [Wilks Lambda $=0.95, F(5,228)=2.20$, $p=0.055$, partial eta squared 0.05]. In the total group, women were more tired over time when they were more neurotic, less agreeable, or more introverted (see Table 2 and Fig. 2). Neuroticism had a large effect size (partial eta squared 0.27). There was no interaction between neuroticism and extraversion $(p>0.05)$. Because the BC and BBP groups did not differ from each other with regard to personality, the women scoring high on fatigue were not only women with BC. In addition, fatigue in low conscientious women changed over time: after an increase at $\mathrm{T} 2$ and $\mathrm{T} 3$, the scores returned to baseline level (see Fig. 3).

The regression analyses showed that before including depressive symptoms into the analysis, neuroticism $(\beta=0.45$, $p<0.001)$ and extraversion $(\beta=-0.27, p<0.05)$ were the only predictors of fatigue at $\mathrm{T} 4$, explaining $48 \%$ of the variance. When depressive symptoms were included in the third step, baseline depressive symptoms $(\beta=0.29, p<0.05)$, neuroti$\operatorname{cism}(\beta=0.29, p<0.05)$, and extraversion $(\beta=-0.25, p<0.05)$ predicted fatigue 6 months later, explaining $52 \%$ of the variance. With baseline fatigue added to the regression equation, only neuroticism $(\beta=0.22, p<0.05)$ and baseline fatigue ( $\beta=0.79, p<0.001)$ predicted fatigue, explaining $70 \%$ of the variance. Demographic characteristics, diagnosis, and adjuvant therapy (chemotherapy, radiotherapy, hormone therapy) did not play a role.

Table 2 Differences in personality characteristics (scoring high or not high on a factor) and the influence on fatigue scores over time

\begin{tabular}{lcccc}
\hline $\begin{array}{l}\text { Personality } \\
\text { characteristics }\end{array}$ & $d f$ & $F$ & $p$ & $\begin{array}{l}\text { Partial eta } \\
\text { squared }\end{array}$ \\
\hline Extraversion & 1 & 5.03 & 0.026 & 0.04 \\
Agreeableness & 1 & 7.59 & 0.007 & 0.05 \\
Conscientiousness & 1 & 3.90 & 0.050 & 0.03 \\
Neuroticism & 1 & 48.76 & 0.000 & 0.27 \\
Openness & 1 & 0.49 & 0.49 & 0.004 \\
\hline
\end{tabular}

\section{Discussion}

The aim of the study was to examine the relationship between personality factors and fatigue in patients having a palpable lump in the breast or being recalled for further examination after an abnormality on a screening mammography. The BC patients feel more tired after treatment had started, but their fatigue levels return to baseline value after a few months. In the total group, low scorers on extraversion and agreeableness and high scorers on neuroticism showed a stable high fatigue level. After controlling for depressive symptoms, neuroticism and extraversion predicted fatigue 6 months later. When baseline fatigue was included in the analysis, extraversion was not a significant predictor anymore. Future research could study underlying mechanisms to generate ideas for interventions. In addition, other fatigue-related factors, such as anxiety and sleep quality, that could mediate the relationship between personality and fatigue, should also get attention.

In line with earlier research, several months after diagnosis, fatigue was mild in severity in a majority of the $\mathrm{BC}$ patients [2]. In line with Smets et al. [26] and Greenberg et al. [13], just after radiation therapy, fatigue was increased but returned quickly to baseline level. This also explains why receiving adjuvant therapy did not predict fatigue 6 months after surgical treatment. It might be that fatigue, like subjective well-being [14], is only temporarily affected by a major life event, such as being diagnosed with and treated for cancer. Another possible explanation is the response-shift phenomenon: patients get used to increased levels of fatigue and reset their internal standards [13, 27].

Personality was the main interest. Our findings not only show that fatigue is quite stable in these women. Personality seems to have a sound influence on the experienced level of fatigue, irrespective of demographic or medical factors or depressive symptoms. As was already found in earlier studies [29, 30], extraversion and neuroticism were predictors of fatigue. Smets et al. [25] suggested that the link between neuroticism and fatigue could be explained by 
Fig. 2 Course of fatigue over 6 months in women scoring low or high on extraversion
Fatigue

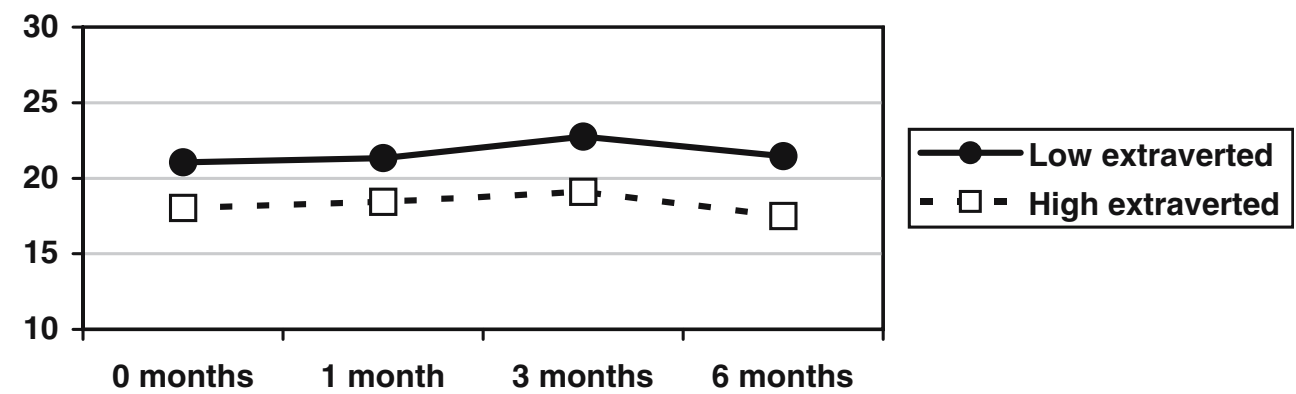

the fact that individuals high in negative affect report more symptoms, probably due to their inner fixation and the tendency to interpret symptoms as threatening. Neuroticism and depressive symptoms were indeed found to be related in several studies [6, 17]. Our findings did not support this idea because after adding baseline depressive symptoms and baseline fatigue, neuroticism remained a predictor. In the study by Sugawara et al. [30], neuroticism was associated with fatigue, independent of depressive symptoms. Different measurement methods of personality and depression, number of measurement points, and type of patients might explain these conflicting findings. Neuroticism is characterized by several constructs, such as anxiety and anger hostility. These various constructs might relate differently to fatigue and depression. Our personality measure could not differentiate between the various components of neuroticism. Future studies could examine this further.

Some explanations for the relationship between extraversion and fatigue have been raised. Extraversion can be partially defined as being energetic. The NEO-FFI extraversion factor consists of several facets, and one of these is labelled activity. Fatigue in cancer patients has often been defined as lack of energy [31]. In contrast, this extraversion facet denotes employing a lot of various daily activities. A low scorer on this facet prefers a more relaxed life style, which does not refer to being unenergetic. Nevertheless, cancer-related fatigue and daily activity are inversely related [1]. Thus, one of the possible connections between extraversion and fatigue might be mediated by potential amount of daily activity. Secondly, another NEO-facet of extraversion-labelled positive emotions is strongly associated with happiness and well-being. Fatigue is a distressing symptom in cancer patients, thereby impairing QOL [26, 29]. Being cheerful might also be the mediating link between extraversion and fatigue.

Baseline fatigue was not only associated with extraversion but also with depressive symptoms. The relationship between depressive symptoms and fatigue has often been found because fatigue is one of the symptoms of depression. However, in the present study, items of the CES-D and the FAS loaded on two different factors (data not shown), as in the development phase of the FAS [19]. This is in line with a study on BC survivors [5], in which fatigue, instead of being secondary to depression, cooccurred with depression as part of a coordinated response elicited by cytokine actions of the central nervous system.

Before diagnosis was known, the $\mathrm{BC}$ group was less tired than the BBP group. Although the $\mathrm{BC}$ group was older than the BBP group and more women from the latter group had paid work, neither age nor having paid work was associated with fatigue scores. Furthermore, fatigue was also unrelated to the other demographic. Results of previous studies are inconsistent at this point. We do not have an explanation for the lower fatigue scores in the $\mathrm{BC}$ patients before diagnosis.

Like demographic factors and adjuvant therapy, diagnosis also did not predict fatigue at $\mathrm{T} 4$. One reason for this
Fig. 3 Course of fatigue over 6 months in women scoring low or high on conscientiousness

\section{Fatigue}

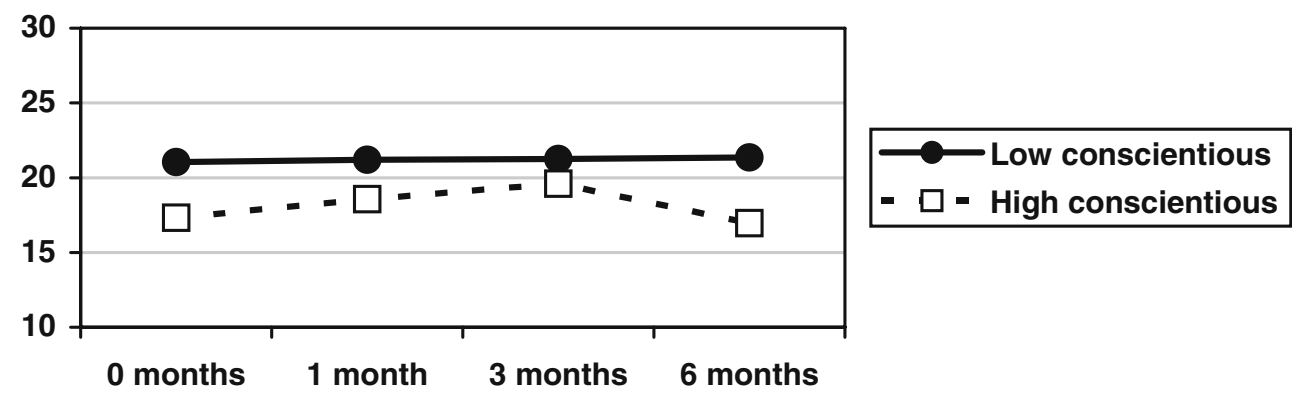


finding might be the fact that fatigue scores at $\mathrm{T} 4$ had returned to baseline level. Another explanation might be that studies reporting that demographic factors, adjuvant therapy, and/or diagnosis explained fatigue scores did not examine personality factors, which appear to play an important role in fatigue. Concerning demographic characteristics, previous studies that included personality also found that age, education level, and/or marital status did not predict fatigue scores, especially when they had to compete with other predictors in a model.

We did not measure fatigue in women with large tumors. This study focussed on women with T1-T3 stage and women with a benign breast disease, with the diagnosis being made after entrance into the study. Therefore, we focussed on women with smaller tumors and not, for instance, on women with T4 stage. We did not intend to examine the differences in fatigue between $\mathrm{BC}$ patients with different stages of diseases. It might well be that fatigue levels are higher in women with large tumors, as for example, these women often have metastases and have chemotherapy for a considerable length of time. We did not examine this, but future research on this topic seems relevant.

One important limitation of the present study is the fact that the FAS has not been validated in cancer patients. However, internal consistency and factorial structure are good, and because in the BBP group there was a high correlation $(r=0.91)$ between fatigue at 3 and 6 months, test-retest reliability also appeared good. Furthermore, the response rate is approximately $62 \%$, which is comparable with other studies [18] that include patients before diagnosis is known. This is probably caused by feelings of uncertainty or stress surrounding the diagnosis. These feelings were also the reason for a number of women for not participating in the study. The dropout rate was higher in the BBP group. The patients who did not complete the study were similar to the patients who remained in the study on any factor. The main reason for dropout in the BBP group was that patients no longer wanted to be confronted with their visit to the hospital. In the $\mathrm{BC}$ group, stress was the main reason for dropping out of the study.

To conclude, personality, especially neuroticism, is more strongly related to fatigue than demographics, the diagnosis cancer, receiving cancer treatment, and baseline depressive symptoms and fatigue. When replicated, screening and treating women who are at risk to experience high levels of fatigue is recommended.

\section{References}

1. Ahlberg K, Ekman T, Gaston-Johansson F, Mock V (2003) Assessment and management of cancer-related fatigue in adults. Lancet 362:640-650
2. Andrykowski MA, Curran SL, Lightner R (1998) Off-treatment fatigue in breast cancer survivors: a controlled comparison. J Behav Med 21:1-18

3. Association of Comprehensive Cancer Centres (1998) Incidence of cancer in The Netherlands. Drukkerij De Kempen, Utrecht, The Netherlands

4. Beekman ATF, Deeg DJH, Van Limbeek J, Braam AW, De Vries MZ, Van Tilburg W (1997) Criterion validity of the Center for Epidemiologic Studies Depression Scale (CES-D): results from a community-based sample of older subjects in The Netherlands. Psychol Med 27:231-235

5. Bower JE, Ganz PA, Aziz N, Fahey JL (2002) Fatigue and proinflammatory cytokine activity in breast cancer survivors. Psychosom Med 64:604-611

6. Cassano P, Fava M (2002) Depression and public health: an overview. J Psychosom Res 53:849-857

7. Cella D, Lai JS, Chang CH, Peterman A, Slavin M (2002) Fatigue in cancer patients compared with fatigue in the general United States population. Cancer 94:528-538

8. Cohen JW (1988) Statistical power analysis for the behavioral sciences, 2nd edn. Lawrence Erlbaum Associates, Hillsdale, NJ

9. Costa PT Jr, McCrae RR (1989) NEO PI/FFI manual supplement. Psychological Assessment Resources, Odessa, FL

10. De Vries J, Michielsen HJ, Van Heck GL, Drent M (2004) Measuring fatigue in sarcoidosis: The Fatigue Assessment Scale (FAS). Br J Health Psychol 9:279-291

11. Digman JM (1990) Personality structure: emergence of the fivefactor model. Annu Rev Psychol 41:417-440

12. Goldberg LR (1990) An alternative 'description of personality': the big-five factor structure. J Pers Soc Psychol 59:1216-1229

13. Greenberg DB, Sawicka J, Eisenthal S, Ross D (1992) Fatigue syndrome due to localized radiation. J Pain Symptom Manage 7:38-45

14. Headey B, Wearing A (1989) Personality, life events, and subjective well-being: toward a dynamic equilibrium model. J Pers Soc Psychol 57:731-739

15. Hoekstra HA, Ormel J, De Fruyt F (1996) Handleiding bij de NEO PersoonlijkheidsVragenlijsten NEO-PI-R en NEO-FFI [Manual for the NEO Personality Inventories NEO-PI-R and NEO-FFI]. Swets \& Zeitlinger, Lisse, The Netherlands

16. Jacobsen PB, Hann DM, Azzarello LM, Horton J, Balducci L, Lyman GH (1999) Fatigue in women receiving adjuvant chemotherapy for breast cancer: characteristics, course, and correlates. J Pain Symptom Manage 18:233-242

17. Jylha P, Isometsa E (2006) The relationship of neuroticism and extraversion to symptoms of anxiety and depression in the general population. Depress Anxiety 23:281-289

18. Lampic C, Thurfjell E, Bergh J, Sjödén P-O (2001) Short- and long-term anxiety and depression in women recalled after breast cancer screening. Eur J Cancer 37:463-469

19. Michielsen HJ, De Vries J, Van Heck GL (2003) Psychometric qualities of a brief self-rated fatigue measure: the Fatigue Assessment Scale (FAS). J Psychosom Res 54:345-352

20. Michielsen HJ, De Vries J, Van Heck GL, Van de Vijver AJR, Sijtsma K (2004) Examination of the dimensionality of fatigue: the construction of the Fatigue Assessment Scale (FAS). Eur J Psychol Assess 20:39-48

21. Michielsen HJ, De Vries J, Drent M, Peros-Golubicic T (2005) Psychometric qualities of the Fatigue Assessment Scale in Croatian sarcoidosis patients. Sarcoidosis Vasc Diff Lung Dis 22:133-138

22. Radloff LS (1977) The CES-D Scale: a self-report depression scale for research in the general population. Appl Psychol Meas 1:385-401

23. Schroevers MJ, Sanderman R, Van Sonderen E, Ranchor AV (2000) The evaluation of the Center for Epidemiologic Studies 
Depression (CES-D) scale: depressed and positive affect in cancer patients and healthy reference subjects. Qual Life Res 9:1015-1029

24. Servaes P, Prins J, Verhagen S, Bleijenberg G (2002) Fatigue after breast cancer and in chronic fatigue syndrome. Similarities and differences. J Psychosom Res 52:453-459

25. Smets EM, Visser MR, Willems-Groot AF, Garssen B, Oldenburger F, van Tienhoven G, De Haes JC (1998) Fatigue and radiotherapy: (a) experience in patients undergoing treatment. $\mathrm{Br} \mathrm{J}$ Cancer 78:899-906

26. Smets EMA, Visser MRM, Garssen B, Frijda NH, Oosterveld P, De Haes JCJM (1998) Understanding the level of fatigue in cancer patients undergoing radiotherapy. J Psychosom Res 45:277-293

27. Sprangers MA, Van Dam FS, Broersen J, Lodder L, Wever L, Visser MR, Oosterveld P, Smets EM (1999) Revealing response shift in longitudinal research on fatigue - the use of the then test approach. Acta Oncol 38:709-718
28. Stone P, Hardy J, Huddart R, A'Hern R, Richards M (2000) Fatigue in patients with prostate cancer receiving hormone therapy. Eur J Cancer 36:1134-1141

29. Stone P, Richards M, A'Hern R, Hardy J (2001) Fatigue in patients with cancers of the breast or prostate undergoing radical radiotherapy. J Pain Symptom Manage 22:1007-1015

30. Sugawara Y, Akechi T, Okuyama T, Matsuoka Y, Nakano T, Inagaki M, Imoto S, Fujimori M, Hosaka T, Uchitomi Y (2005) Occurrence of fatigue and associated factors in disease-free breast cancer patients without depression. Support Care Cancer 13:628636

31. Winningham ML (2001) Strategies for managing cancer-related fatigue syndrome: a rehabilitation approach. Cancer 92:988-997

32. Young KE, White CA (2006) The prevalence and moderators of fatigue in people who have been successfully treated for cancer. J Psychosom Res 60:29-38 